\title{
Transverse Incision for the Management of Infected Non-Unions of Tibia
}

\author{
Mussa Wardak ${ }^{1}$, Emal Wardak $^{2}$, Ajmal Wardak $^{3}$ \\ ${ }^{1}$ Department of Orthopaedics, Al-Haj Professer Mussa Wardak Hospital, Kabul, Afghanistan \\ ${ }^{2}$ Wazir Akbar khan Goverment Hospital, Kabul, Afghanistan \\ ${ }^{3}$ Mussa Wardak Hospital, Kabul, Afghanistan \\ Email: emalpgi@gmail.com
}

Received August 10, 2011; revised October 20, 2011; accepted December 20, 2011

\begin{abstract}
We are describing a transverse incision that we have found very useful in performing surgical procedures where the fractured site needs freshening, excision of the necrotic bones or shortening. Operative procedures using this technique has been extensively used by us since 1980 and we have registered 1187 cases with average follow up of 10 years. The incision is transverse and can be performed in any part of leg and extends from $1 \mathrm{~cm}$ lateral to anterior border of the tibia and runs medially till $1 \mathrm{~cm}$ medial to medial border of tibia. The incision allows adequate visualization of the interested area, less of periosteal stripping, ease of closure, no post operative wound dehiscence and cosmetically very much acceptable.
\end{abstract}

Keywords: Transverse Incision; Infected Nonunions; Ilizarov

\section{Introduction}

Longitudinal incision in extremities are the standard incisions when we perform our daily surgical procedures, whether for fracture fixation, tumor excision, debridement etc., this is because longitudinal incisions have the advantage of being more anatomical, we don't cross many zones in one section so we preserve the blood supply distally as well as sensations. But if we look at the anatomy of leg the antero-medial surface of the leg is covered only by skin and no significant neurovascular structure cross this area.

In our practice open compound fractures of tibia which are mainly due to warfare injuries have got infection and non union rate of as high as $40-50 \%$ [1], and while performing debridement, sequestrectomies and bone excisions using the longitudinal incision we found that we compromise the vascularity of the area by dissecting the periosteoum much more than required, and in areas we excise the necrotic fragments and create shortening, closure of the longitudinal incision is difficult and higher incision breakdowns [2]. So we thought of using transverse incision instead, and since 1980 we are using this incision we have used it on 1187 cases, which are operated using this technique and we have experienced no notable complications. We searched the literature and found no literature describing such an incision except few just mentioning about it [2-4].

\section{Materials and Method}

We started using transverse incision to see if we can minimize incision related complication in warfare injuries, infected nonunions of tibia and pseudo-arthrosis of tibia since 1980 . We have registered 1187 cases, which were operated using this technique and the number increases day by day. Their age ranges from 8-70 yrs old of them in 286 cases the transverse incision technique was performed primarily, meaning at the time of first debridement and fixation and the rest are the cases which were either had infected nonunions (40.1\%) and/or pseudoarthrosis (59.9\%). The area of involvement was $15 \%$ in upper third, $65 \%$ in middle third and $25 \%$ in the lower third of tibia.

\section{Operative Technique}

Transverse incision is to be performed only in anteromedial part of the leg. Meaning in that part of the leg where it's only covered with a thin layer of soft tissue. In this region there are no main or any important neurovascular structures except the Saphenous vein which is usually sacrificed.

The starting point is $1 \mathrm{~cm}$ lateral to anterior border of the tibia and extends till medial border of the tibia if needed it can be extended up to $2 \mathrm{~cm}$ more towards medial side (Figure 1), so in this way we get 8-10 cm transverse incision. 

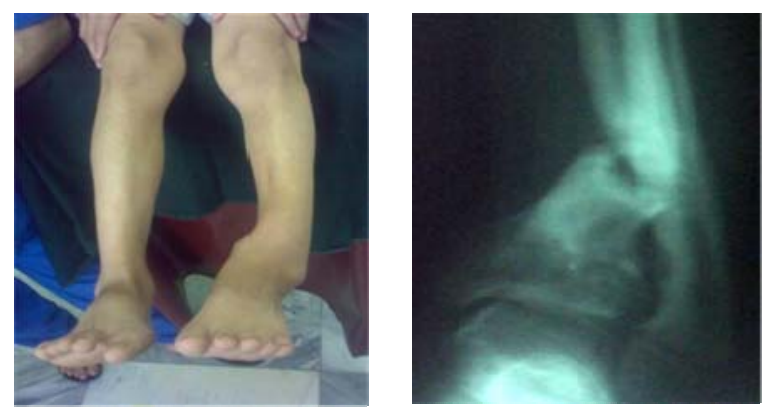

Figure 1. Clinical and radiological pictures of Pseudo-arthrosis of tibia in a 14-year old boy.

In the lower third of the tibia be careful not to cut the tendon of tibialis anterior muscle, after the incision is given mobilize the incision and put retractors distally and then reach the bone and dissect it sub-periosteally, perform the desired procedure if you want to see more of the area you can do that by asking the assistant to put two right angled retractors one on each end medio-laterally to increase your field of vision. our aim should be to minimize trauma to surrounding soft tissue and perisoteoum as they are already compromised and we use this incision mainly for this purpose if we are not gentle with our dissection it will eliminate the advantages of transverse incision, as we have already mentioned that this incision is meant for infected nonunions and pseudo-arthrosis of the tibia and freshening of the bony ends can be done (Figure 2).

In cases of infected nonunions where there is extensive involvement of the bone, resection of the dead necrotic bone can be achieved using radiological evidence and proper preoperative planning and using the percutaneous Gigli saw technique 1, this will make the procedure less traumatic, less time consuming and less of periosteal stripping.

\section{Results and Discussion}

This transverse incision is based on a thorough study of the anatomy of this area of the leg, including its neurovascular and lymphatic supply. The obvious goal of a good surgical approach is to provide adequate exposure of the pathologically involved structures while at the same time minimizing morbidity resulting from damage to the blood vessels, nerves and tendons in the area.

This incision is adequate enough so that the surgeon is not hindered in performing a procedure on any portion of the operative field and all pathological involvement is easily visualized (Figure 3).

The transverse incision allows the surgeon to correct a deformity in all planes simultaneously and allows clear visualization of the anatomical structures while doing so. Satisfactory reduction and stabilization of the tibia is easily accomplished under direct vision (Figure 4).

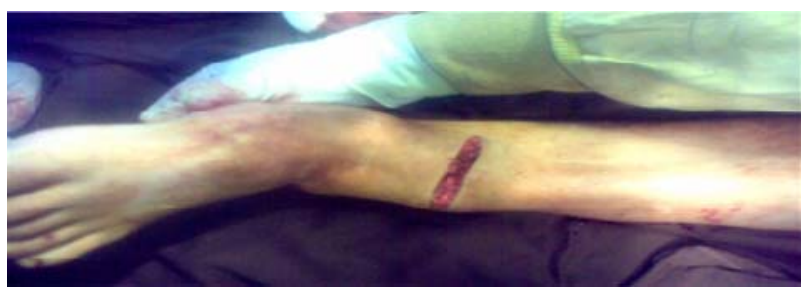

Figure 2. Transverse incision which is around $8 \mathrm{cms}$.

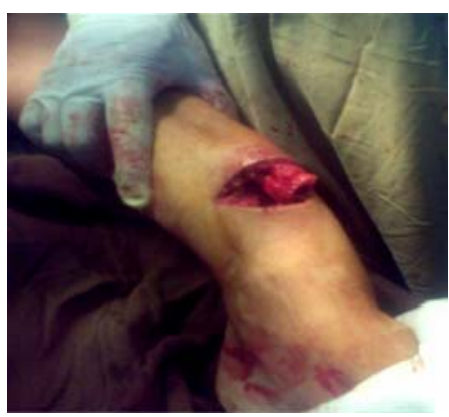

Figure 3. Desired bonny procedures can be performed very adequately.

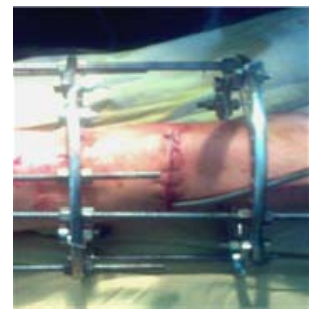

Figure 4. Skin closure is easy and no tension on the skin edges.

This incision has also proved to be more acceptable cosmetically than are alternative incisions, as it often heals with only a barely perceptible scar (Figure 5).

The problems that may be associated with other operative approaches including tender and cosmetically unacceptable scars and incision dehiscence have not been a problem with this incision (Figure 6).

Problems with this incision are negligible. Limited exposure is not a problem if our incision is placed on the right place where pathology is there, the only drawback of this incision is that it can't be extended proximally and or distally. Patients who had previous surgical procedures done on the same or opposite limb using longitudinal incision were very satisfied with the appearance of the scar using transverse incision. This was particularly so when the parents could compare this scar with that resulting from a longitudinal incision.

At the end we would like to recommend this incision for War injuries where there is fragment loss and acute shortening is required.

Infected nonunions where there is need for resection of dead or sequestra is required. 


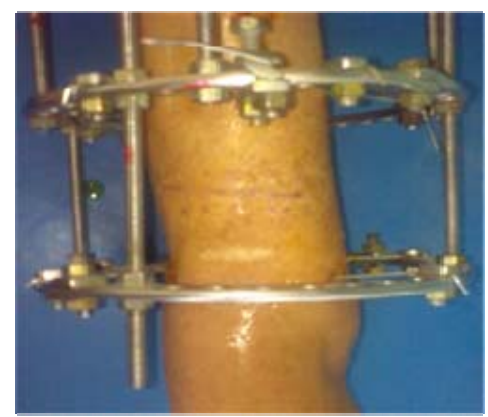

Figure 5. Would after suture removal.
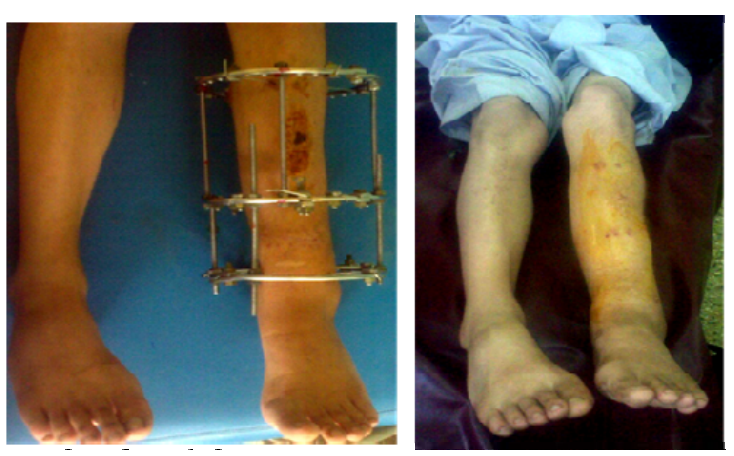

Figure 6. Limb of the patient during and after the treatment with a well healed and cosmetic scar.
Pseudo-arthrosis of tibia.

Fracture reduction where open intramedullary nailing is done in select cases.

Proper patient selection and good dissection will make this incision the incision of your choice once you start using.

\section{REFERENCES}

[1] M. M. Wardak and A. R. Seyawash, "Role of Extrafocal Osteosynthesis in Modern Orthopaedics and Traumatolgy,” Academy of Sciences of Republic of Afghanistan, Kabul, 1989.

[2] M. P. Magadum, C. M. Basavaraj Yadav, M. S. Phaneesha and L. J. Ramesh, "Acute Compression and Lengthening by the Ilizarov Technique for Infected Nonunion of the Tibia with Large Bone Defects," Journal of Orthopaedic Surgery, Vol. 14, No. 3, 2006, pp. 273-279.

[3] A. H. R. W. Simpson, C. Andrews and H. Giele, "Skin Closure after Acute Shortening, Journal of Bone and Joint Surgery, Vol. 83, No. 5, 2001, pp. 668-671. doi:10.1302/0301-620X.83B5.11585

[4] J. L. Marxen and D. L. Osterfeld, "The Cincinnati Incision: A Comprehensive Approach for Surgical Procedures of the Foot and Ankle in Childhood," Journal of Bone and Joint Surgery, Vol. 64, No. 9, 1982, pp. 1355-1358. 